\title{
THE EFFECT OF INSULIN ON THE GLUCOSE TOLERANCE OF NORMAL MAN
}

\author{
By J. D. ROSENBAUM, H. DeKRUIF, 1 and P. H. LAVIETES \\ (From the Department of Internal Medicine, Yale University School of Medicine, New Haven)
}

(Received for publication May 5, 1943)

\section{INTRODUCTION}

Utilization of carbohydrate by the diabetic patient probably differs only quantitatively from that of the normal subject. Many factors which influence carbohydrate tolerance in normal subjects (for example, exercise, emotional disturbances, infections, and the nature of the preceding diet) do likewise in diabetic patients. Any measure which affects the tolerance of the normal subject for carbohydrate merits investigation for its applicability to the treatment of diabetes.

The administration of insulin to non-diabetic subjects may provoke diabetic responses to glucose tolerance tests. This has been observed in obese (1), undernourished $(2,3)$, and psychotic (4) patients. In the present experiments, in an attempt to elucidate this phenomenon, the effect of small doses of crystalline and protamine insulin on the respiratory metabolism, as well as on the conventional glucose tolerance test, of normal man has been investigated.

\section{EXPERIMENTAL PLAN AND PROCEDURES}

Normal male subjects, maintained on constant diets moderately restricted in carbohydrate, were given small doses of crystalline or protamine insulin for periods of 2 to 6 days. The response of the blood sugar and respiratory quotient to the ingestion of carbohydrate was determined in the morning, before, during, and after the insulin period. The insulin was always given after completion of the day's test.

The authors served as subjects for these experiments. They also made all the analyses except for some of the blood sugar determinations which were made by the technical staff, under the direction of Pauline $M$. Hald. In addition, they carried on their usual clinical and teaching responsibilities. The subjects avoided strenuous exercise throughout the studies, so that the caloric expenditure must have been fairly constant from day to day; some variation was, of course, unavoidable. Diets were calculated in household measures in the first 3 experiments, by weight in the fourth. In the first 2 experiments, the body weight fell slightly; in the last 2 experiments, a more liberal caloric intake prevented loss of weight.

${ }_{1}^{1}$ This article represents work done in fulfilment of the thesis requirement for the degree of Doctor of Medicine at Yale University School of Medicine.
Fifty grams of glucose in $200 \mathrm{cc}$. of water, flavored with lemon juice, was used as a test dose, replacing breakfast. The subjects avoided unnecessary activity before coming to the laboratory, where they rested in the reclining position for at least one-half hour before the observations were begun. Oxalated blood samples were taken before, and 90 and/or 120 minutes after, the glucose. In the first 2 experiments, 0.2 cc. of capillary blood was used; in the last two, 2 cc. of venous blood. Protein-free filtrates were prepared within 30 minutes of sampling, by the method of Somogyi (5), for determination of blood sugar in duplicate according to Benedict (6). Duplicate determinations agree within 3 per cent, with rare exceptions. The fasting and post-glucose specimens were analyzed simultaneously, so that comparisons between the two are probably subject to an error no greater than this. Absolute values are less accurate, since, in some instances, the dilute standard solutions were observed to have deteriorated. In control experiments, the blood sugar returned consistently to or below the fasting (or, more properly, post-absorptive) level, 2 hours after the ingestion of the glucose.

Respiratory quotients were determined by standard techniques (7), in the fasting (post-absorptive) state, and 50 and 80 minutes after the glucose. Samples were received in the Tissot apparatus over 10-minute periods; the times refer to the mid-point of these periods. The subjects were all trained in breathing through valves. All gas analyses were made in duplicate, with agreement within 0.03 per cent. Analyses of atmospheric air were run as a further check on technique. In a control experiment, in which water was taken instead of glucose, there was no significant change in either the respiratory quotient or the blood sugar. After at least 30 minutes of rest in the recumbent position, the subject breathed through the valves for 3 minutes before the collection of gas was begun. In each experiment, duplicate basal gas collections were made several times, the first after 30 to 40 minutes and the second after 51 to 66 minutes of rest. The results, presented in Table I, indicate that the respiratory quotient reached a constant level in one-half hour in experiments 1,2 , and 3 but not in experiment 4 . The respiratory data of the first experiment, therefore, cannot be interpreted, and will be presented only for those days on which 2 preliminary runs were made.

\section{RESULTS}

Before presenting the data for the individual experiments, a preliminary appraisal of the respiratory data is necessary. The increases of 
TABLE I

Respiratory quotient

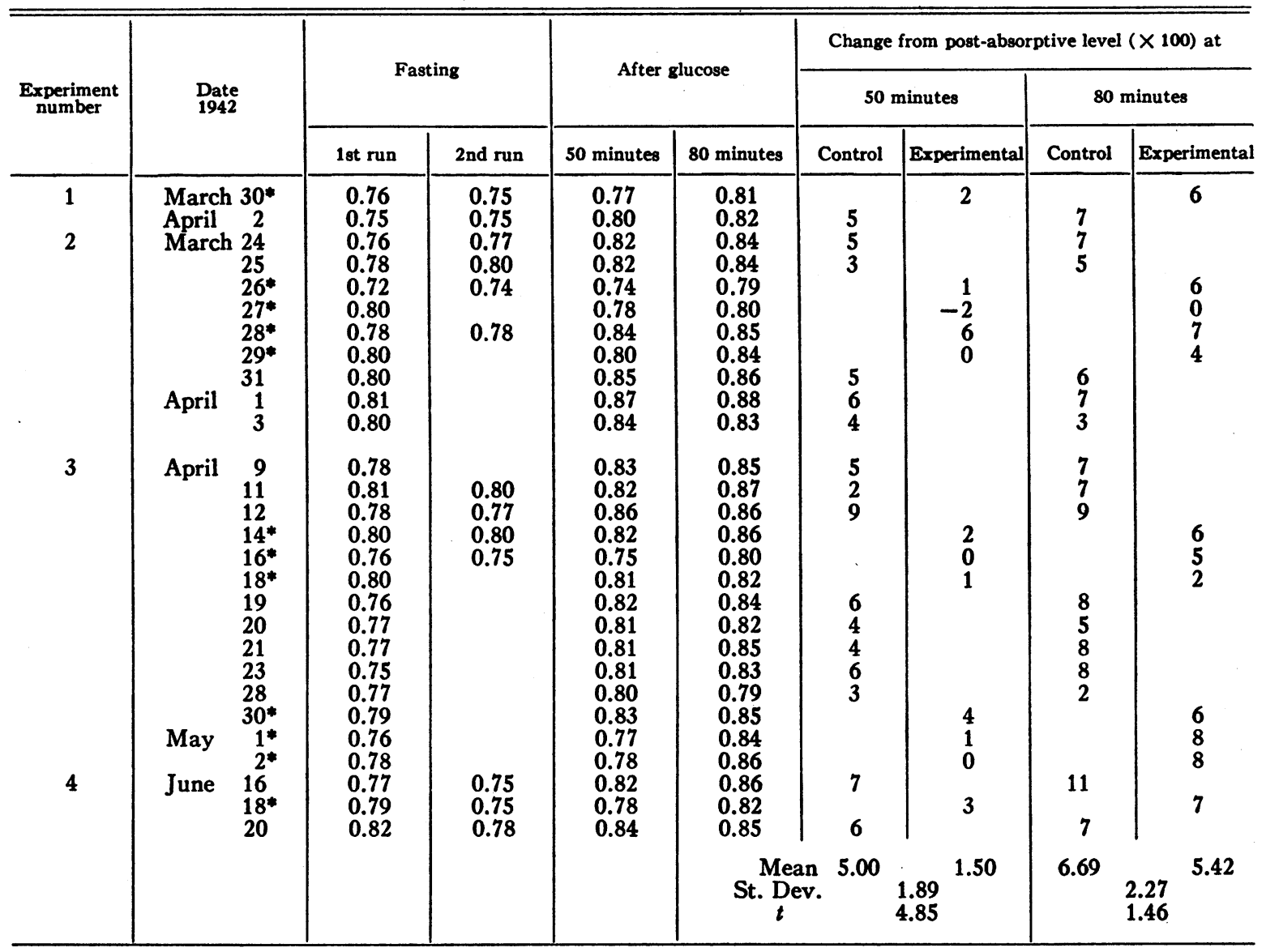

* Days following insulin administration.

R.Q. over the fasting level at 50 and 80 minutes after glucose ingestion are presented in the last 4 columns of Table I. The experimental observations are those in which insulin has been given during the preceding 24 hours. During $50 \mathrm{~min}$ utes after the ingestion of glucose, the mean rise in R.Q. is 0.050 in the control group, 0.015 in the experimental one. Statistical treatment indicates that the difference between these values is highly significant. At 80 minutes, the mean rise is 0.067 in the control group, 0.054 in the experimental one. The difference in these means is probably significant only in that it could have occurred by chance in 1 of 5 trials. It is evident that the rise of R.Q. after glucose arrives at or near a maximum within 80 minutes in all of our experiments, though the rise is probably slightly smaller when insulin has been given on the pre- ceding day. The rise is tardy in the latter instance, however, as demonstrated by the highly significant differences observed at 50 minutes. Since the control and experimental observations approach one another in the 30 -minute interval between observations, it is not surprising that there is some overlapping of results at 50 minutes.

The data of the indivdual experiments will be presented graphically, omitting, for the sake of simplicity, the values for R.Q. 80 minutes after glucose. In each of 4 pre-insulin control periods of experiment 1 (Figure 1), the blood sugar 90 minutes after 50 grams of glucose per os falls below the fasting level. When 20 units of protamine insulin are given daily, the blood sugar 90 minutes after the glucose is significantly higher than the fasting level. This effect is still present 87 hours after the last injection; a feeding at 
bedtime prevents it. Oxidation of the ingested glucose begins less promptly on the morning after the last dose of insulin than it does 3 days later.

In 2 control studies in experiment 2 (Figure $2)$, the blood sugar 90 minutes after glucose is slightly greater than the post-absorptive level. The difference becomes sharply increased, however, when protamine insulin is given, and remains so 63 hours after the last dose of insulin. The evening and bedtime feedings have no clearcut effect on the hyperglycemia in this experiment. In 2 control experiments before starting insulin, the R.Q. rises 0.05 and 0.03 , respectively, in the 50 minutes after the administration of glucose. When 25 units of protamine insulin are given daily, the corresponding rises are $0.01,0.02$, 0.06 , and 0.00 . Night feedings result in normal oxidative responses to glucose on the following morning, despite insulin. The oxidative response is also normal 63 hours after the last injection of insulin, though the glucose still provokes excessive hyperglycemia at that time.

In experiments 3 and 4 , venous blood was analyzed before and 2 hours after 50 grams of

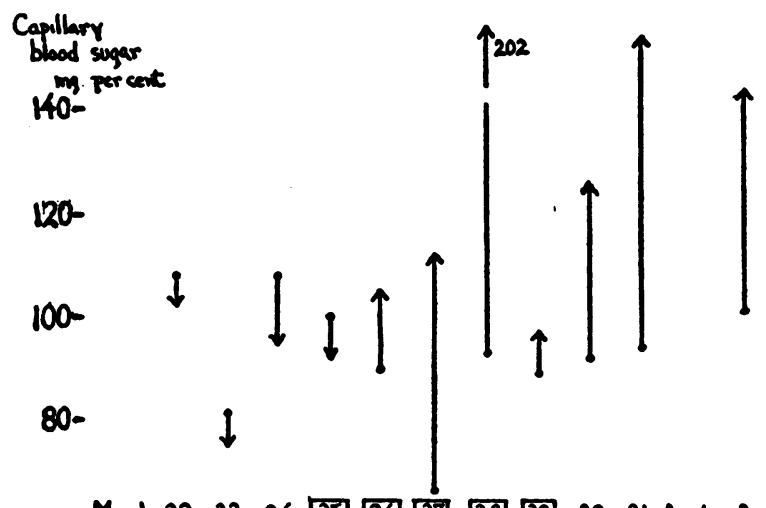

Respinatory
quetents
79
77
$78-$

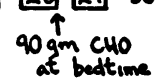

Fig. 1. EXPERIMENT 1

Subject: DeK. Diet P. 80, F. 105, C. 160, including 50 grams glucose at 8 A.M. and $200 \mathrm{cc}$. of milk at 10:30 A.M. Protamine insulin, $U$ 20, taken in afternoon of days designated by squares about dates. No frank hypoglycemic episodes. Dots represent post-absorptive blood sugar and R.Q. Arrow heads mark values for blood sugar 90 minutes, and R.Q. 50 minutes after 50 grams of glucose per os.

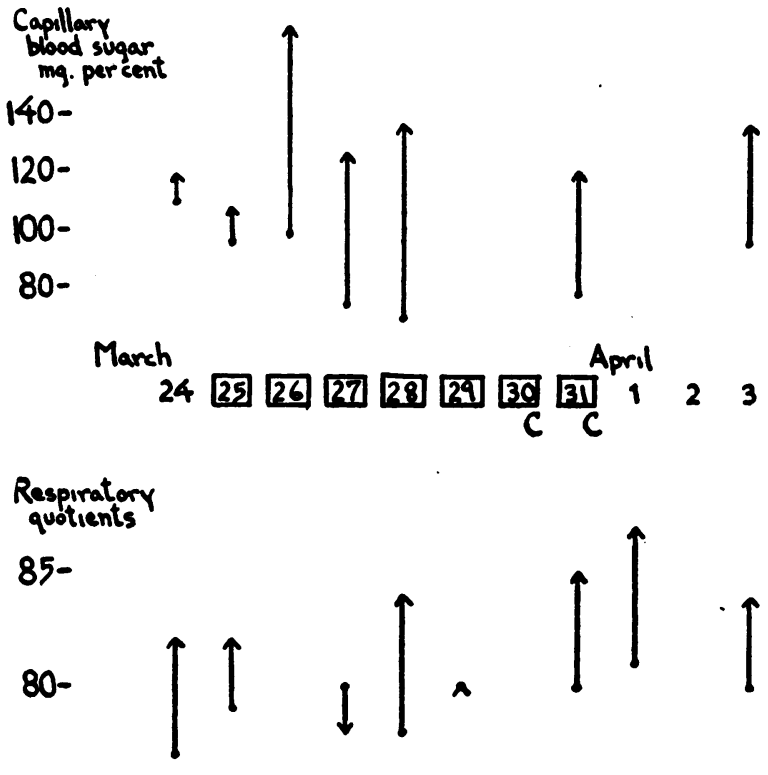

$75-$

\section{$\uparrow$}

Fig. 2. EXPERTMENT 2

Subject: P. H. L. Diet P. 90, F. 115, C. 220, started on March 21. Protamine insulin, U 25, taken on afternoons of days designated by squares about dates. Mild sweating and restlessness during night of March 25; otherwise no hypoglycemic symptoms. 80 grams of carbohydrate taken in divided doses during the evening of March 30, and 100 grams at bedtime on March 31 . Symbols as in Figure 1.

glucose per os. This is a conventional test, the normal response to which is a return to or below the original level in 2 hours. Since, in some of our insulin periods, the fasting level is well below that of the control periods, we interpret responses as abnormal only if the 2 -hour blood sugar is higher than both the fasting level of the same day and the highest 2-hour level of the control period. With such rigid criteria, even a single abnormal response during the insulin periods becomes significant.

In experiment 3 (Figure 3 ), 3 control studies were made before each trial of insulin. In none of these did the blood sugar exceed $83 \mathrm{mgm}$. per cent 2 hours after glucose, and in each instance this was lower than the fasting level. Four of the 8 observations after insulin were abnormal by our criteria, the 2-hour blood sugar being 117 and 112 on 2 occasions. Blood sugars were determined at 90 minutes as well as 120 minutes in 


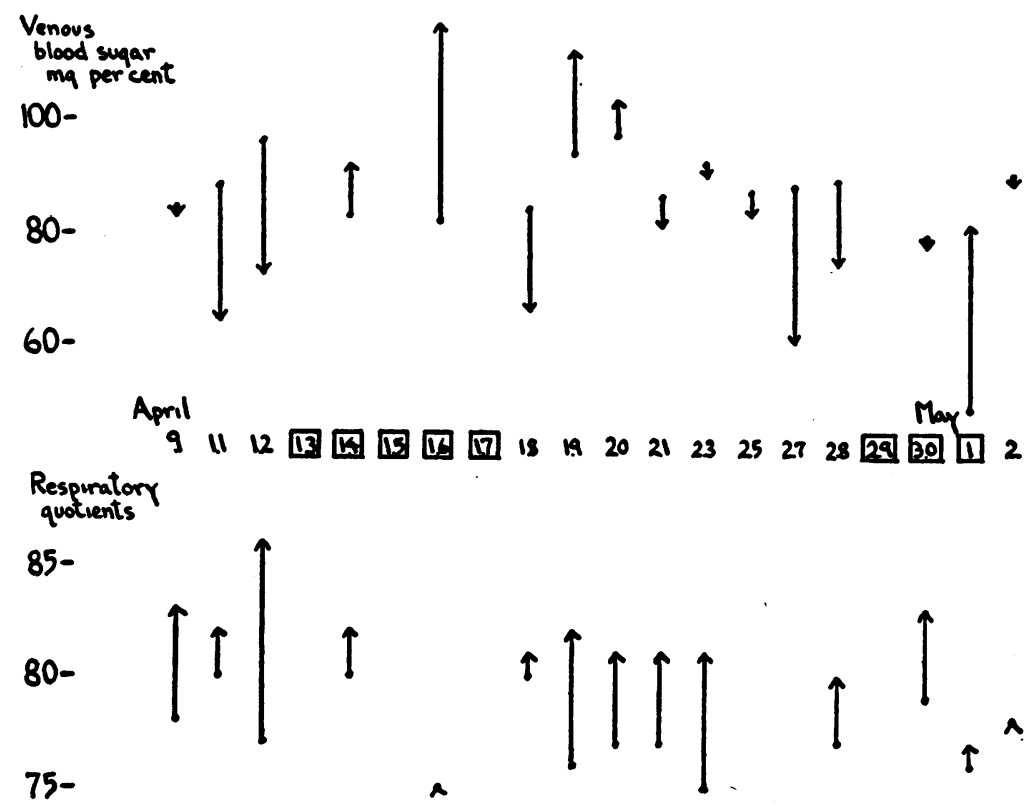

Fig. 3. Experiment 3

Subject: J. D. R. Diet P. 95, F. 135, C. 175, including P. 8, F. 17, C. 17 in mid-afternoon, started April 7. Protamine insulin, U 15, given each afternoon April 13 to 17 inclusive, and U 221/2, 25, and 15 respectively on April 29, 30 , and May 1, respectively. One frank mild shock on arriving in laboratory on morning of May 1. Dots represent post-absorptive blood sugars and R.Q.'s. Arrow heads indicate blood sugar 120 minutes, and R.Q. 50 minutes, after 50 grams of glucose per os.

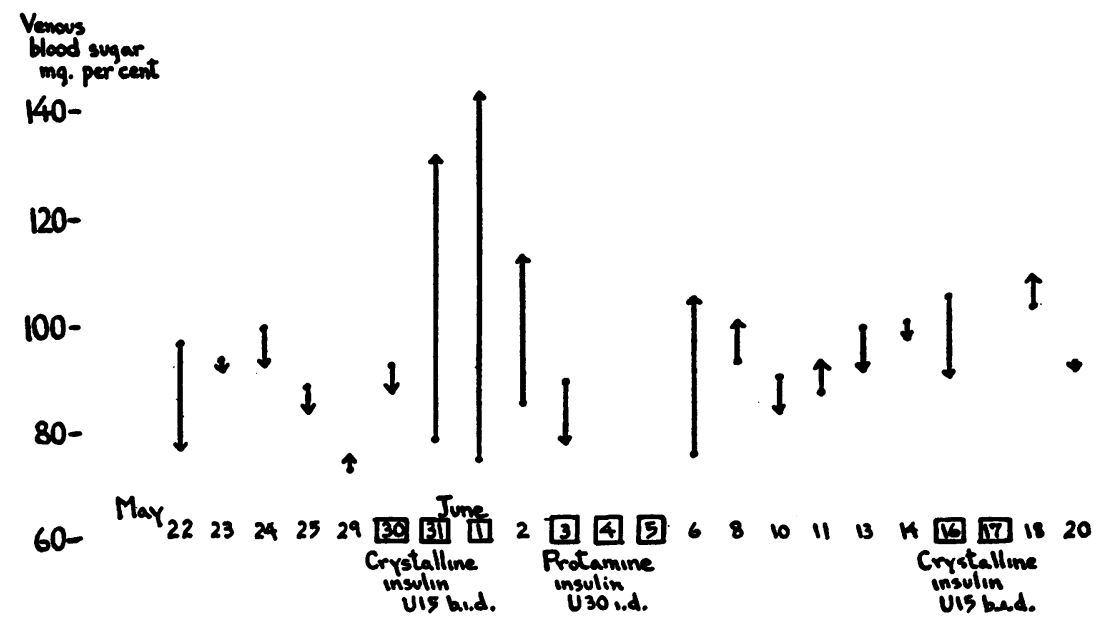

Fig. 4. Experiment 4

Subject: P. H. L. Diet P. 100, F. 170, C. 230, including 50 grams glucose at 8:00 A.M., P. 13, F. 19, C. 22 at 10:30 A.M., and P. 9, F. 11, C. 22 at 9:00 P.M., started May 19. Crystalline insulin was given at 10:15 A.M. and 5:45 P.M. It provoked mild sweating and tremor just before lunch regularly, and at bedtime occasionally. Protamine insulin, given in the afternoon, induced no hypoglycemic symptoms. Dots and arrow heads as in Figure 3. 
this experiment, but since the former parallel the more conventional 2 -hour values, they are omitted in the interest of simplicity of presentation. The rise of R.Q. at 50 minutes is $0.05,0.02$, and 0.09 before the first course of insulin; $0.02,0.00,0.01$ during it; and $0.06,39$ hours after the last dose. With the second course of insulin, the R.Q. fails to rise with normal promptness in 2 or 3 studies. The R.Q. is low during the hypoglycemic episode of May 1, and fails to rise within 50 minutes of the ingestion of the glucose test dose.

In all of 10 control observations in experiment 4 (Figure 4), the 2-hour blood sugar is below $100 \mathrm{mgm}$. per cent and is lower than the fasting level. This is true in none of the 5 observations of the insulin periods, the 2-hour blood sugar reaching 132 and 144 on 2 occasions. Normal responses return within 36 hours after crystalline insulin, but not within 63 hours after the last dose of protamine insulin. The R.Q. rose only 0.03 in the 50 minutes after glucose administration on June 18, following a trial of crystalline insulin. Control observations on June 16 and 20 show rises of 0.07 and 0.06 , respectively (Table $\mathrm{I}$ ).

\section{DISCUSSION}

Since insulin can produce delay in oxidation of carbohydrate in normal subjects on fairly liberal diets, a similar effect in patients with diabetes seems not improbable. This is in keeping with clinical observations that the tolerance of such patients for carbohydrate may be sharply reduced by over-dosage with insulin. Our experiments emphasize the importance of avoiding over-dosage, and of supplying carbohydrate at the times when insulin action is maximal. This is usually in the mid-morning when regular insulin is used before breakfast, and in the evening when it is used before supper. They clearly indicate a need for a bedtime feeding when protamine insulin is used at any time of the day, even though symptoms of hypoglycemia during the night may be lacking.

The mechanism by which insulin reduces tolerance for carbohydrate is not apparent. One possibility is suppression of the insulin-secreting mechanism of the pancreas. There is, indeed, some evidence that the insulin content of the pancreas falls when insulin is given (8). An alternative hypothesis is that insulin, by stimulating oxidation of carbohydrates, reduces that available for formation of liver glycogen (9), establishing a condition comparable to the so-called "starvation diabetes" (10). The two hypotheses are not mutually exclusive since suppression of pancreatic insulin has been produced by restriction of carbohydrate intake (11), as well as by administration of insulin. The occurrence of frank hypoglycemia in experiment 3 , when the respiratory quotient is only 0.76 , indicating only slight combustion of carbohydrate, suggests that the readily available glycogen stores are depleted, as they are in starvation. The analogy with starvation diabetes is further supported by the observation that the ketones of post-absorptive blood samples were unequivocally elevated on two occasions during the insulin periods. In experiment 1 (March 28), these were $7.1 \mathrm{mgm}$. per cent, and, in experiment 3 (May 1), $3.2 \mathrm{mgm}$. per cent, as contrasted with control levels of 2.2 and $1.7 \mathrm{mgm}$. per cent, respectively, on the same diet without insulin. These determinations were made by Dr. Bernard L. Kartin by the method of Somogyi (12).

Failure of the glucose tolerance curve to return to normal within three days of stopping protamine insulin can only be attributed to continued action of protamine insulin over this period. If protamine insulin had a less durable action, it would be difficult to explain the cumulative effect which makes the time of administration of a daily dose of protamine insulin to diabetic patients immaterial (13). The relative speed with which the oxidative response to glucose reverts to normal after stopping insulin is disturbing, but a similar difference in the speed of recovery of normal oxidative response and of hyperglycemic response to ingested glucose has been observed after starvation (14).

\section{SUMMARY AND CONCLUSIONS}

1. In normal man, under controlled conditions, small doses of insulin result in excessive hyperglycemia and delayed rise of respiratory quotient after ingestion of glucose. Both effects are usually evident within less than 24 hours of the start of insulin administration, and both are reversible, the delay in oxidation more promptly than the excessive hyperglycemia. Extra feedings at bedtime prevent loss of tolerance on the following morning. 
2. An analogy is drawn between the above effects and "starvation diabetes."

3. The clinical significance of these observations is discussed.

\section{BIBLIOGRAPHY}

1. Wilder, R. M., Smith, F. H., and Sandiford, I., Observations on obesity. Ann. Int. Med., 1932, 6, 724.

2. Maher, J. T., and Somogyi, M., Effect of insulin on carbohydrate tolerance of non-diabetic individuals. Proc. Soc. Exper. Biol. and Med., 1938, 37, 615.

3. Blotner, H., Late results following use of insulin in 100 cases of malnutrition. New Eng. J. Med., 1938, 218, 371.

4. Appel, J. W., and Hughes, J., The effect of large doses of insulin on glucose tolerance. Am. J. M. Sc., 1940, 199, 829.

5. Somogyi, M., A method for the preparation of blood filtrates for the determination of sugar. J. Biol. Chem., 1930, 86, 655.

6. Benedict, S. R., The determination of blood sugar. II. J. Biol. Chem., 1928, 76, 457.
7. Peters, J. P., and Van Slyke, D. D., Quantitative Clinical Chemistry. Vol. II. Methods. Williams and Wilkins, Baltimore, 1932.

8. Best, C. H., and Haist, R. E., The effect of insulin administration on the insulin content of the pancreas. J. Physiol., 1941, 100, 142.

9. Cori, C. F., and Cori, G. T., The fate of sugar in the animal body. VIII. The influence of insulin on the utilization of glucose, fructose, and dihydroxyacetone. J. Biol. Chem., 1928, 76, 755.

10. Chambers, W. H., Undernutrition and carbohydrate metabolism. Physiol. Rev., 1938, 18, 248.

11. Best, C. H., Haist, R. E., and Ridout, J. H., Diet and the insulin content of the pancreas. J. Physiol., 1939, 97, 107.

12. Weichselbaum, T. E., and Somogyi, M., A method for the determination of small amounts of ketone bodies. J. Biol. Chem., 1941, 140, 5.

13. Mark, M. F., Optimum time for administration of protamine zinc insulin. Arch. Int. Med., 1939. 64, 897.

14. Dann, M., and Chambers, W. H., Animal calorimetry. The metabolism of glucose administered to the fasting dog. J. Biol. Chem., 1930, 89, 675. 